11.4;01.3

\title{
Фазовая кластеризация в иерархическом ансамбле связанных осцилляторов ван дер Поля
}

\author{
(C) O.C. Темная ${ }^{1,2}$, G.P. Tsironis ${ }^{2}$ \\ ${ }^{1}$ Институт радиотехники и электроники им. В.А. Котельникова РАН, Москва, Россия \\ ${ }^{2}$ Национальный исследовательский технологический университет „МИСиС“, Москва, Россия \\ E-mail: arnellemorte@gmail.com
}

Поступило в Редакцию 24 сентября 2020 г.

В окончательной редакции 23 января 2021 r.

Принято к публикации 3 фревраля 2021 г.

\begin{abstract}
Исследовано образование фазовых кластеров в иерархическом ансамбле связанных осцилляторов ван дер Поля. Показано, что иерархически организованный ансамбль в динамическом режиме демонстрирует образование фазовых кластеров на каждом уровне иерархии, причем это сопровождается кластеризацией частот, соответствующих спектру нормальных мод. Показано, что кластеризация не разрушается в случае неидентичности собственных частот осцилляторов и случайного распределения их начальных фаз. В состоянии кластеризации одинаковыми значениями фаз обладают элементы ансамбля, находящиеся на одном уровне иерархии.
\end{abstract}

Ключевые слова: осциллятор ван дер Поля, синхронизация, кластеризация, иерархия.

DOI: 10.21883/PJTF.2021.09.50900.18561

Динамика сетей взаимосвязанных элементов исследуется уже несколько десятилетий, однако эта задача остается крайне важной для ряда дисциплин, таких как физика, радиотехника, биология, химия, энергетика, информационные технологии и социальные науки. Среди наиболее важных динамических процессов в этих средах и структурах выделяют синхронизацию, кластеризацию и образование пространственно-временны́х структур (химеры, бризеры, солитоны). Значение результатов, получаемых благодаря анализу осцилляторной динамики сложных сетей, сложно переоценить. Например, в сентябре 2003 г. в Италии произошел полный обрыв сети Интернет, который спровоцировал прекращение работы всех электростанций города, в том числе снабжающих электроэнергией больницы [1]. Обрыв происходил лавинообразно, начавшись на локальной энергетической станции. Одной из причин возникшей ситуации является существование тупиковых ветвей сетей, возникающих при проведении коммуникаций на новые территории $[1,2]$. Другой пример касается изучения состояния головного мозга и когнитивных процессов [3]. Анализ когерентности разных ритмов позволяет обнаружить нарушения работы мозга, благодаря чему терапию можно назначать намного раньше [4].

Изучение встречающихся в природе и технике сетей представляет собой нетривиальную задачу из-за их сложности, исходящей от топологии и динамики связей и элементов. В настоящее время активно изучаются адаптивные динамические сети, для которых характерные времена изменения топологий и динамических характеристик узлов приблизительно одинаковы [5]. Тем не менее для некоторых сетей влияние статической структуры на возникновение различных нелинейных явлений изучено недостаточно. Настоящая работа является продолжением исследования иерархических ансамблей, первой частью которого стал анализ спектров нормальных мод в древовидных сетях [6]. Актуальность рассмотрения этих систем заключается в их широкой распространенности: от белковых соединений и структуры головного мозга до энергетических сетей и нетрадиционных источников энергии [7]. Установлено, что в простейшей иерархической сети взаимосвязанных осцилляторов в виде бинарного дерева спектр нормальных колебаний имеет форму фрактала „чертова лестница“. Следующим шагом является исследование такой сети в режиме незатухающих колебаний на примере ансамбля нелинейных осцилляторов. Ранее [8] вопрос о динамике сетей нелинейных осцилляторов изучался на примере осциллятора ван дер Поля как осциллятора с предельным циклом, который демонстрирует простейшую бифуркацию Андронова-Хопфа и применяется для моделирования сложных биологических процессов. Цель настоящей работы - исследование фазовой кластеризации в иерархическом ансамбле взаимосвязанных осцилляторов ван дер Поля.

Рассмотрим нелинейную динамику ансамбля иерархически связанных идентичных осцилляторов ван дер Поля (рис. 1), реализованных в виде параллельных колебательных контуров $L G C$ с нелинейными активными элементами (АЭ), взаимосвязанных через индуктивности. Мы предполагаем для простоты консервативный характер связи (через индуктивности). Вольт-амперная характеристика АЭ каждого $j$-го осциллятора в рабочей точке имеет вид

$$
i_{j}\left(u_{j}\right)=G_{1} u_{j}+G_{3} u_{j}^{3},
$$

где $u_{j}(j=1, \ldots, N)$ - мгновенное напряжение на контуре; параметры $G_{1,3}$ выбраны так, что $G_{1}<0, G_{3}>0$; 


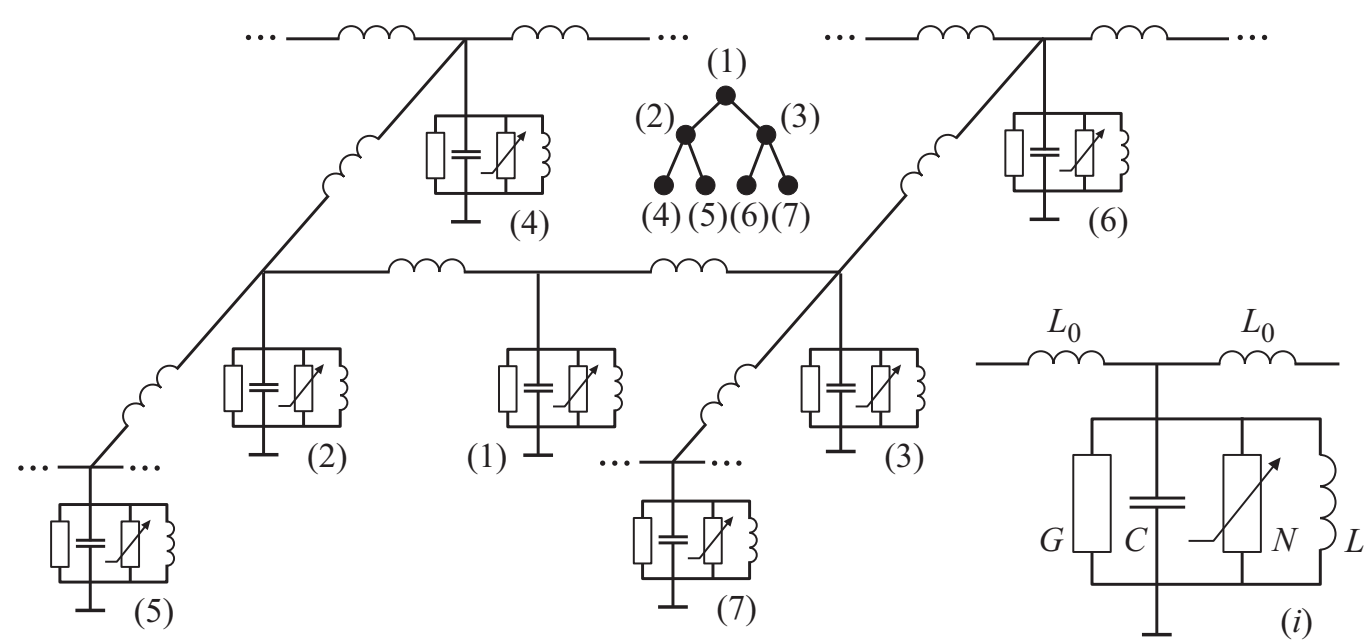

Рис. 1. Иерархический ансамбль взаимосвязанных осцилляторов ван дер Поля. На вставке изображен $L G C$-контур с нелинейным АЭ $N$ и индуктивностями связи.

$N$ - количество осцилляторов в ансамбле. Систему дифференциальных уравнений для определения напряжений $u_{j}$ легко записать исходя из законов Кирхгофа. Пропуская промежуточные выкладки, имеем

$$
\begin{gathered}
\frac{d^{2} u_{j}}{d t^{2}}-\frac{\left|G_{1}\right|-G}{C}\left(1-\frac{3 G_{3}}{\left|G_{1}\right|-G} u_{j}^{2}\right) \frac{d u_{j}}{d t} \\
+\frac{1}{L C} u_{j}=\frac{1}{L_{0} C} \sum_{k=1}^{N} a_{j k} u_{k},
\end{gathered}
$$

где $a_{j k}$ - элементы матрицы связей $k$-го элемента с $j$-м, $L_{0}, L, C, G$ - индуктивность связи, индуктивность, емкость и проводимость отдельных осцилляторов ван дер Поля соответственно. Отметим, что матрица связей $a_{j k}$ является блочно-иерархической [9] и каждый осциллятор, кроме первого, связан с двумя осцилляторами более высокого уровня иерархии и одним осциллятором более низкого.

Введем нормированные амплитуды $x_{j}$ и время $\tau$ следующим образом:

$$
x_{j}=\frac{u_{j}}{\sqrt{\left(\left|G_{1}\right|-G\right) / 3 G_{3}}}, \quad \tau=\omega_{0} t .
$$

Здесь $\omega_{0}=\frac{1}{\sqrt{L C}}-$ собственная частота колебательной системы осциллятора (считаем все осцилляторы идентичными по параметрам). С учетом (2) дифференциальное уравнение (1) примет вид

$$
\frac{d^{2} x_{j}}{d \tau^{2}}-\varepsilon\left(1-x_{j}^{2}\right) \frac{d x_{j}}{d \tau}+x_{j}=\alpha \sum_{k=1}^{N} a_{j k} x_{k},
$$

где $\varepsilon=\left(\left|G_{1}\right|-G\right) / \rho-$ параметр самовозбуждения осциллятора, $\rho=\sqrt{L / C}-$ характеристическое сопротивление контуров, $\alpha=L / L_{0}-$ нормированный параметр связи. При $\alpha=0$ уравнение (3) описывает хорошо известный единичный осциллятор ван дер Поля [10], в котором режим самовозбуждения автоколебаний реализуется при $\varepsilon>0$. Рассмотрим динамику модели (3) при вариации параметра связи $\alpha$ и фиксированном значении $\varepsilon$ (до режима релаксационных колебаний). Свойства иерархической системы (3) при $\varepsilon=0$ (линейный случай) ранее изучались в работе [6].

На рис. 2, а представлена трехмерная карта временны́х реализаций $x_{j}(\tau)$ на плоскости номер осциллятора-время для количества осцилляторов $N=32$, a на рис. 2,b- осциллограммы напряжений $x_{5}(\tau)$, $x_{10}(\tau), x_{25}(\tau)$, демонстрирующие временну́ю динамику в соседних кластерах. Нумерация осцилляторов представлена на рис. 1 , при моделировании использовались следующие параметры: $\alpha=0.5, \varepsilon=0.1, N=32$. Параметры модели выбраны так, чтобы каждый осциллятор находился в автоколебательном режиме с достаточно сильной связью между осцилляторами. Видно, что на каждом из уровней иерархии происходит кластеризация напряжений $x_{j}(\tau)$ по фазе, причем на соседних уровнях иерархии колебания происходят в противофазе. Помимо фазовой кластеризации на каждом уровне иерархии проявляется также частотная кластеризация. Частоты колебаний на каждом уровне иерархии соответствуют спектру нормальных частот.

На рис. 3 показано образование фазовых кластеров в ансамбле иерархически связанных осцилляторов при случайном распределении начальных напряжений $(a)$ и собственных частот $(b)$ на контурах. Видно, что фазовая кластеризация сохраняется и в этих случаях, однако не сохраняется равенство фаз на каждом кластере. В моделировании предполагался равномерный закон распределения начальных значений фаз/собственных частот на интервале $[0,1]$. Таким образом, реализация иерархической сети сохраняет свойства кластерности фаз колебаний даже при неидентичности параметров или случайном распределении начальных условий. Также было проведено численное моделирование, которое 
продемонстрировало наличие критического параметра связи $\alpha$, при котором происходит образование фазовых кластеров. В частности, при $\alpha=0$ кластеризация отсутствует, с увеличением $\alpha$ растут размах спектра нормальных мод колебаний и размеры фазовых кластеров.

Таким образом, в работе исследовано образование фазовых кластеров в иерархически связанном ансамбле осцилляторов ван дер Поля. Показано, что иерархическая организация ансамбля в динамическом режиме демонстрирует образование фазовых кластеров на каждом уровне иерархии, причем это сопровождается кластеризацией частот, соответствующих спектру нормальных мод. Показано, что кластеризация не разрушается в случае неидентичности собственных частот осцилляторов и случайного распределения их начальных фаз. В состоянии кластеризации одинаковыми значениями фаз обладают элементы ансамбля, находящиеся на одном уровне иерархии. Предлагаемый анализ может быть проведен и для другого типа связи (емкостного, резистивного). Можно ожидать при этом аналогичного эффекта фазо-
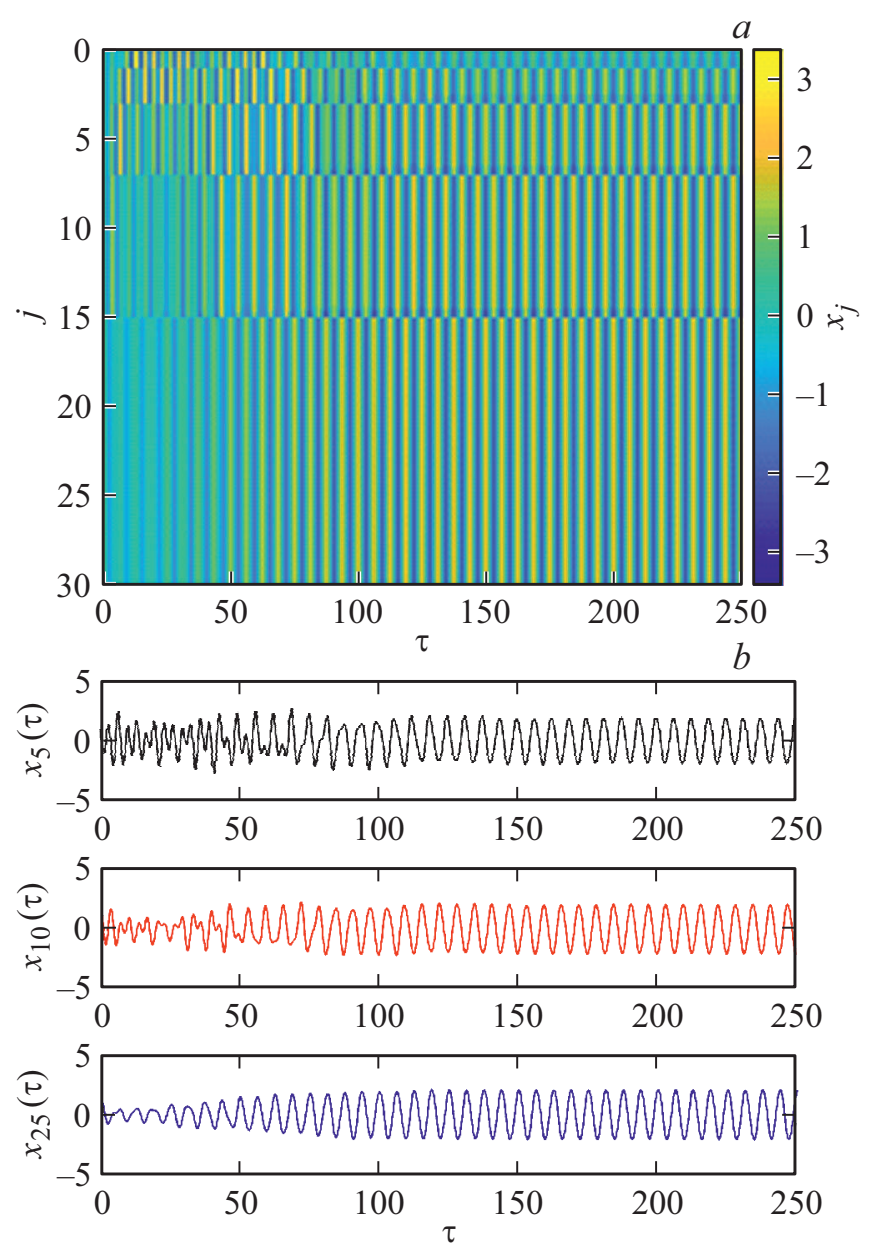

Рис. 2. $a-$ карта кластеризации напряжений $x_{j}(\tau)$ в ансамбле иерархических осцилляторов ван дер Поля на плоскости номер осциллятора-время; $b-$ осциллограммы напряжений $x_{5}(\tau), x_{10}(\tau), x_{25}(\tau)$, демонстрирующие временну́ю динамику в соседних кластерах. Параметры моделирования: $\alpha=0.5$, $\varepsilon=0.1, N=32$.
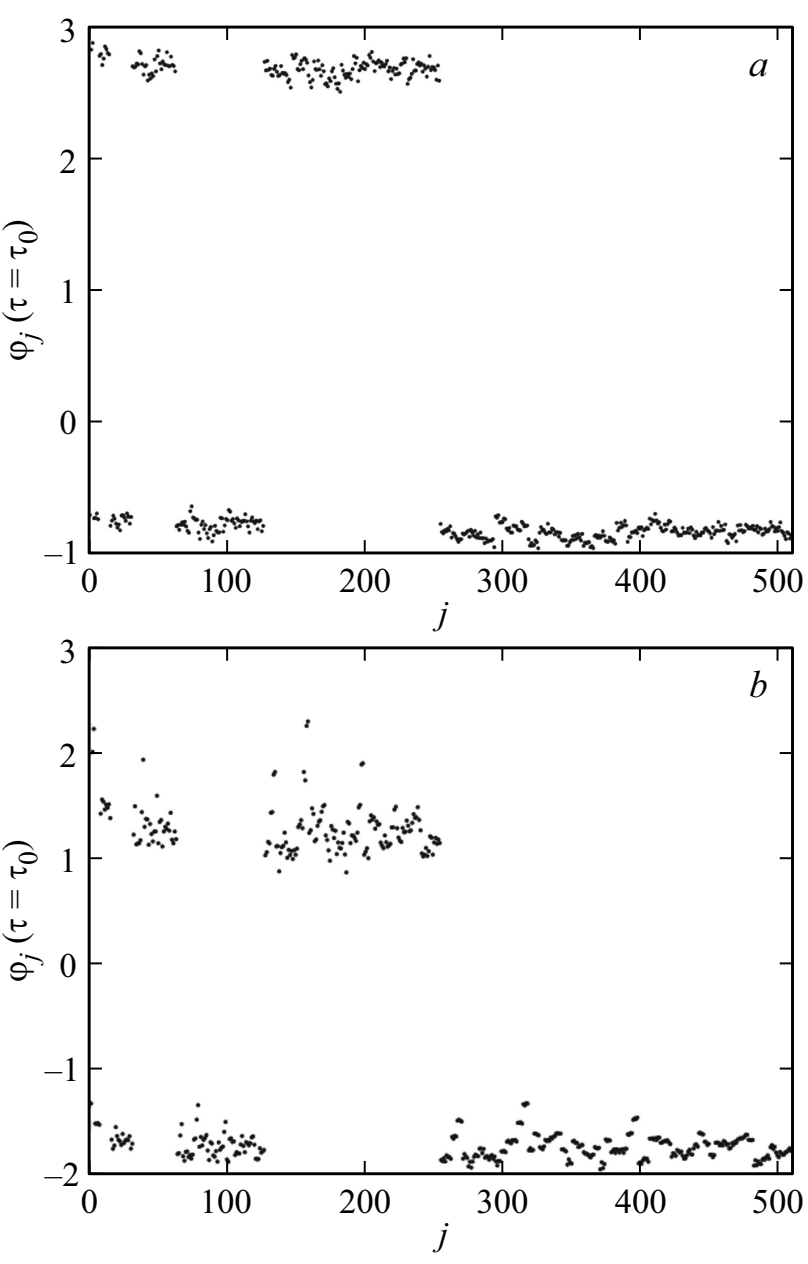

Рис. 3. Распределение текущих фаз осцилляторов $\varphi_{j}$ от времени $\tau$ в ансамбле иерархически связанных осцилляторов при случайном распределении начальных напряжений на контурах $(a)$ и случайном распределении собственных частот $(b)$. Количество осцилляторов $N=512, \tau_{0}=250-$ максимальное время интегрирования системы.

вой кластеризации на разных уровнях иерархии. Проведенное исследование может быть использовано для создания сетей наноразмерных осцилляторов $[11,12]$ в применении, например, к нейроморфным вычислениям, где вопрос топологии организации ансамбля играет ключевую роль.

\section{Финансирование работы}

Работа выполнена при финансовой поддержке грантов Российского фонда фундаментальных исследований № 19-29-03015, 18-29-27018 мк, частичной поддержке Министерства науки и образования РФ в рамках Программы повышения конкурентоспособности НИТУ „МИСиС“ (грант № К2-2019-010), а также государственного задания РФ № 0030-2019-0013. 


\section{Конфликт интересов}

Авторы заявляют, что у них нет конфликта интересов.

\section{Список литературы}

[1] S.V. Buldyrev, R. Parshani, G. Paul, H.E. Stanley, S. Havlin, Nature, 464, 1025 (2010). DOI: 10.1038/nature08932

[2] P.J. Menck, J. Heitzig, J. Kurths, H.J. Schellnhuber, Nature Commun., 5, 3969 (2014).

DOI: doi.org/10.1038/ncomms4969

[3] F. Siebenhühner, S.H. Wang, J.M. Palva, S. Palva, eLife, 5, e13451 (2004). DOI: 10.7554/eLife.13451

[4] V.F. Kitchigina, Front. Integr. Neurosci., 27 (12), 12 (2018). DOI: $10.3389 /$ fnint.2018.00036

[5] О.В. Масленников, В.И. Некоркин, УФН, 187 (2), 745 (2017). DOI: $10.3367 / \mathrm{UFNr} .2016 .10 .037902$

[6] О.С. Сафина, А.В. Воронов, А.Р. Сафин, М.Ф. Булатов, Д.В. Чуриков, Е.Д. Суровяткина, Письма в ЖТФ, 45 (17), 24 (2019). DOI: 10.21883/PJTF.2019.17.48219.17651

[7] M. Fang, G. Dong, R. Wei, J.C. Ho, Adv. Energy Mater., 7 (23), 1700559 (2017). DOI: 10.1002/aenm.201700559

[8] D.A. Linkens, IEEE Proc. A, 130 (2), 69 (1983).

[9] R. Rammal, G. Toulouse, M. Virasoro, Rev. Mod. Phys., 58 (3), 765 (1986). DOI: 10.1103/RevModPhys.58.765

[10] А.П. Кузнецов, Е.С. Селиверстова, Д.И. Трубецков, Л.В. Тюрюкина, Изв. вузов. Прикладная нелинейная динамика, 22 (4), 3 (2014).

[11] A.R. Safin, N.N. Udalov, M.V. Kapranov, Eur. Phys. J. Appl. Phys, 67 (2), 20601 (2014). DOI: 10.1051/epjap/2014130518

[12] M. Zahedinejad, A.A. Awad, S. Muralidhar, R. Khymyn, H. Fulara, H. Mazraati, M. Dvornik, J. Åkerman, Nature Nanotechnol., 15 (1), 47 (2020). 